\title{
Analyses of PHP Dynamic Website Development Technology in the Era of Internet +
}

\author{
WU Yan-xia, Jiang man-li \\ Institute of humanities, Jiangxi University of Traditional Chinese Medicine, Nan \\ chang,330004, China
}

Keywords: Internet + era; PHP dynamic website; Development technology

\begin{abstract}
In the development of network dynamic website, PHP technology is comparatively more safe and convenient. In application, it is widely applied to update the stability of dynamic information. Through effective application operation, it can ensure effective information embedding, so as to realize the safety of running the program. In the process of information use, the operation flow of information, compared with the traditional form of writing, is to more effectively promote the effective development of its information. This article discusses PHP dynamic website development technology in the era of Internet + .

In traditional dynamic website development, separate establishment form of programming is vulnerable, at the same time; the development of the script has no unified design standard, which is prone to bugs. The emergence of PHP will adjust the existing situation, and can be more effective to supplement the vulnerability. Based on the original single from code, realize the safety protection of the dynamic website. Below we briefly analyze the development and technical requirements of existing PHP dynamic website development technology.
\end{abstract}

\section{PHP APPLICATION FEATURES}

What is PHP? PHP is the acronym of Hypertext Preprocessor, which is a cross-platform server, embedded scripting language, and has open source. From the form of PHP, it belongs to a submersible HTML form. On page script comments, give $C$ and Perl language development tools, and combined with its own characteristics, mainly run in Web development. A big advantage for PHP application is that it is relatively simple for beginners, and also can provide corresponding advanced features for professional programmers. PHP application can easily develop Web, and also can be used for dynamic website developing. In the form development, PHP is free software construction, and operation is more convenient compared with ASP and JSP. On the development of successful cases of original consciousness, Wikipedia sets huge programming entry. The set of all infrastructure programming is more in line with the existing network base station. And it also has some shortcomings, which make the existing website system not be able to comply with the editor of a large network.

\section{PHP PROJECT EXECUTION PROCESS}

PHP application principle is based on Web network server. By engine editing, realize its implementation on executive function. Execution process of the work is as follows.

First, the user inputs request on the Web browser. According to the suffix expression forms, request analysis, and on the basis of transfer request, execute the request. Based on PHP and Web transmission, write all the file form.

Second, on Web server requests acceptance route, according to its suffix, judge information so as to realize memory information order in hard disk, and on the basis of existing access programming, realize PHP engine application process.

Third, after scanning program files, realize the effective implementation of its data.

Fourth, through engine sound field corresponding web page, feedback to corresponding information customers, and perform information through the server expression form. 


\section{PHP APPLICATION ADVANTAGES}

The editing of modern network technology mainly involves many aspects, other than the development of other application forms; it also needs to carry out effective information set from the actual situation of the web page using technology. The advantages are as follows.

First, it is cross-platform. In the use of PHP, the display of information is through multiple platform, and the application mode of its operation is relatively more abundant.

Second, an easy introduction, and its developing is faster. PHP language has a good compatibility with HTML, and users in the process of operation use Web application specifications. There is the need to make effective measures of information application from PHP command code. According to the existing development environment, the construction of such approaches is also more in line with the existing language construction style.

Third, the source code is higher open, which is in line with the openness of the free application, and the permissions received set is accord with source code use features.

Fourth, enforced rate is higher and it can run faster, so it consumes relatively less resource.

Fifth, in view of more widely database supporting, it can be applied in many modes of development.

Compared with ASP and JSP function, client browser application, without adding more software support environment, should conduct a comprehensive engine analysis according to the code's mixed applications to ensure the execution ability of source code, and ensure that environment application conforms to server requirements. The use of engine execution is more in line with the use of the corresponding standard compared to code operation application.

In theoretical system comparison, the distributed new system is also more in line with the network project. The main hierarchy is three levels: core, business, and expression. In actual application, it may be more levels. Only then, it is able to ensure the integrity reasonable distribution of the overall system, so as to realize the integrity management of the system.

The database connection system is for groups' information applications. With high performance database operations processes, realize data operation fully developed form, and display model core technology with the application of a new generation of data access pattern to realize overall connection on the display. At the same time, compiled database shall guarantee function relation in connection of the whole system, and promote page development conforming to the corresponding database standard. In the application of ASP information run, realize information arrangement by unit information application environment, and according to performance operation requirements, achieve the overall information integrity. And compared to other techniques, its running time units are shown in Table 1 below.

Table 1 The operation comparison of three kinds of dynamic technologies

\begin{tabular}{clll}
\hline Test & ASP & PHP & JSP \\
\hline Cycle operation & 63 & 84 & Without test \\
System operation & 73 & 69 & 13 \\
\hline
\end{tabular}

In different applications, running effect still has certain differences, but from the information above, JSP environment efficiency, compared with other information, is a little bit high. Its server's internal effective concatenated codes should also have access efficiency basis for the comprehensive compilation use, and the rate should be consistent with the existing short message application.

On the security, the information security of PHP, relative to other forms, is a bit safer, because it is between different programming procedures, and the use of specific operation process can also be through different testing methods for the whole task.

\section{PHP DYNAMIC WEBSITE DEVELOPMENT AND DESIGN}

A. Dynamic page design

PHP file is a HTML document which can execute script on the server side, and can generate HTML language with scripts execution by Web server. Itself is part of the original language in web 
pages, and is on the front end, and adopts PHP to implement file name end. PHP files in the process of designing are composed by four parts, which are respectively: (1) HTML tags, HTML tags can be applied in all of its structure design; (2) PHP language command, which is the code in $<$ ? PHP? > tags; (3) include function, include () statement is used in other codes in server applications to realize programming flexibility; (4) scripting language, itself has PHP, C language support function to improve the functional programming.

In PHP file design, tsble and HTML language can be used to display the data got in the database browser. By using SQL, carry on the query to the data in database records, and show the data in Table object later. In PHP file interface design, visualization tools such as Dream Weaver can be applied in implementation. In Web Server, after processed various user request, send the results to a browser which is advantageous for user query.

\section{B. Database access and connection design}

When the user requests through PHP home page, the server will be corresponding to it, and call PHP to execute the file, as well as explain the performed scripts. When there is script database access request, implement database connection through the corresponding function, and its related operation is completed. Later in the process, PHP file also can produce HTML code in accordance with the database access results, and transfer it to the client. In the process, the user cannot see a series of PHP code, but HTML pages, and can significantly improve use security of system.

C. PHP dynamic web applications

In dynamic web design process, we often use methods: IDC (Internet Database Connector), CGI (Common Gateway Interlace) and ADO (ActiveX Data Object). Among them, CGI is simple to use, and in the process of application, execution speed is faster, and disk space and system resources are relatively small. CGI and PHP files are combined in application process, which can effectively realize dynamic web site, and is able to provide certain convenient website management and maintenance. The combination of the two applications is the core technology in the course of dynamic website construction, so it can provide the conditions for PHP technology development and application.

In this paper, in accordance with different browsing time, dynamically display PHP program for different web pages. In case study, we can clearly see PHP application flexibility and convenience.

Add_kf.PHP

$<$ heml $>$

$<$ head $>$

$<$ title $>$ add service personnel $<$ totle $>$

$<$ meta http-equiv $=$ " contene-type" content=" text/heml;

Charest=UTF-8" >

$<$ link type $=$ " text/css" rel=" stylesbeet" href $=$ " style_aft.css" $>$

$<$ head $>$

$<$ body $>$

$<$ ?PHP

If(isset( \$_POST[" submit]))[

Include(" connect.PHP" );

$\$$ aql=" select*from $\mathrm{kf}^{\prime \prime}$;

\$ result=mysql_query( $\$$ aql, $\$$ mysql_id);

$\$$ id=mysql_mum_rows $(\$$ result $)+1$;

Mysql_free_result( $\$$ result);

\$ name_yq= \$_POST[" name_yq" ];

\$ link_yq=\$_POST[" link_yq" ];

\$ aql=" insert into kf(id,kfry,kfxm)valuse(" \$id" , "

\$ name_yq" , " . \$ link_yq. " ) " ;

If( $\$$ result0 \{ 
echo" $<$ hl $>$ operation success $</$ hl $>$ " ;

\}

Else echo" $<$ hl $>$ operation failure $</$ hl $>$ " ;

\section{CONCLUSION}

Along with the rapid development of modern society, people's life cannot be broken up with Web network. In the application of Internet technology, PHP dynamic website development technology has become a mainstream operation. In this paper, such a development technology is used to analysis the main body technical form to provide the corresponding reference for peers.

\section{REFERENCES}

[1] Mu Xingfu, Meng Jun, Yao Xinkui. The construction research of horses information management system under the background of mobile Internet [J]. Journal of Modern Animal Husbandry, 2013,32:29-31.

[2] Li Jing. PHP technique application in small and medium-sized enterprise website development [J]. Computer Knowledge and Technology, 2014:4028-4029 + 4037.

[3] Wang Zhaohua, Wang Haibo. Web page design and facture course reform research in mobile Internet era [J]. Journal of Computer Education, 2013,09:82-86.

[4] Zhang Chaoyang, Xiong Shuhua, Heng Li. The website design and implementation based on Zend Framework [J]. Computer Technology and Development, 2011, 11:197-200.

[5] bing-bing liu, Chen Fei. A kind of new agricultural e-commerce site development method based on osCommerce [J]. Journal of anhui agricultural sciences, 2010,01:247-249.

[6] more than the west king. Dynamic web database connection technology [J]. Journal of tianjin finance and trade management cadre institute, 2010,01:41-43.

[7] Zhao Fu, Ding Xuewei. Based on the Internet platform of mass collaborative innovation research [J]. China soft science, 2009, 12:63-72. 